\title{
rSac3, a new member of Sac domain phosphoinositide phosphatases family
}

\author{
Lijun $\mathrm{Li}^{1,2,3}$, Qi Wan ${ }^{1,2,3}$ \\ ${ }^{1}$ Division of Fundamental Neurobiology, Toronto Western Research Institute, University Health Network, Toronto, Canada M5T 2S8; \\ ${ }^{2}$ Department of Physiology, ${ }^{3}$ Department of Surgery, University of Toronto, Toronto, Canada M5S 1A8.qi.wan@utoronto.ca
}

Cell Research (2007) 17:901-903. doi: 10.1038/cr.2007.94; published online 15 November 2007

Inositol phospholipids are concentrated in the cytosolic surface of membranes. Phosphatidylinositol (PtdIns), the precursor of phosphoinositides, is synthesized primarily in the endoplasmic reticulum. Reversible phosphorylation in the inositol ring of PtdIns at positions 3, 4 and 5 results in the generation of seven phosphoinositide species: PtdIns(3)P, PtdIns(4)P, PtdIns(5)P, PtdIns(3,4)P2, PtdIns(3,5)P2, PtdIns(4,5)P2, PtdIns(3,4,5)P3. Phosphoinositides can be rapidly interconverted from one species to another by strategically localized kinases and phosphatases [1]. Phosphoinositides play a fundamental role in controlling membrane-cytosol interfaces [2]. They are essential regulators of many cellular functions, including classical signal transduction, membrane trafficking, cytoskeletal organization, nuclear events, cell survival versus apoptosis, motility and the permeability and transport of membranes $[1,3]$.

Phosphoinositides are phosphorylated and dephosphorylated by kinases and phosphatases, respectively. One of the most important phosphoinositide kinase families is the phosphoinositide 3-kinase (PI3K), which plays a key role in cell growth, survival, transformation, actin rearrangement, vesicle trafficking, and transcriptional regulation $[1,3]$. Up to nine members of the PI3K family have been reported in mammalian cells [3]. They are grouped into three classes according to the molecules that they preferentially utilize as substrates. Four different lipid products, PtdIns(3)P, PtdIns(3,4)P2, PtdIns(3,5)P2, PtdIns(3,4,5)P3 are generated by PI3K.

Phosphoinositide phosphatases and inositol polyphosphate phosphatases are traditionally classified into four groups based on the position of the phosphate that they hydrolyze, namely 1-, 3-, 4-, or 5-phosphatase [4]. For example, PTEN, the protein product of a tumor suppressor gene, acts as a 3-phosphatase for phosphoinositides and inositol polyphosphates [5]. PTEN dominantly hydrolyzes PtdIns(3,4,5)P3 and thereby keeps it at low levels in vivo. Thus, loss of PTEN function causes an increase of PtdIns(3,4,5)P3 concentration and hyperactivation of the cell-survival protein kinase Akt/PKB (a downstream target of PI3K), which may lead to cell overgrowth and tumor development [3]. Synaptojanin 1 and 2, belonging to the group of 5-phosphatases, participate in synaptic vesicle trafficking through PtdIns $(4,5) \mathrm{P} 2$ hydrolysis. Synaptojanins contain two phosphatase domains. One domain is common to inositol 5-phosphatase, which hydrolyzes PtdIns(4,5)P2 to PtdIns(4)P, but is unable to hydrolyze PtdIns(3)P, PtdIns(4)P, and PtdIns(3,5)P2. The second phosphatase domain of synaptojanins is the Sac domain in N-terminal, which is homologous to yeast Sac1. Sac domain, which marks a novel class of phosphoinositide phosphatases, was first identified in yeast. This domain is a 400 amino acid region that has seven highly conserved motifs, which is thought to be the catalytic and regulatory regions of the phosphatases [4]. The sequence RXNCXDCLDRTN within the sixth highly conserved motif is proposed to be the catalytic core of the Sac domain phosphatases. The CX5R $(\mathrm{T} / \mathrm{S})$ motif found within this sequence is also present in many metal-independent protein and inositide polyphosphate phosphatases. The Sac domain-containing phosphatase is capable of hydrolyzing Ptdins(3)P, PtdIns(4)P and PtdIns(3,5)P2, but not PtdIns(4,5)P2 [4].

In addition to synaptojanins, the Sac domain was subsequently found in several other proteins. Based on the features of the amino acid sequences, the Sac domaincontaining proteins have been divided into two classes [4]. The first class includes human synaptojanin 1 and 2 and the related yeast proteins Inp51p, Inp52p and Inp53p. The Sac 
domain is linked to a C-terminal localized 5-phosphatase catalytic domain and a proline-rich region. The second class of Sac domain proteins includes yeast Sac1p, Fig4p and human KIAA0581 (hSac1), KIAA0966 (hSac2) and KIAA0274 (hSac3). The Sac domain in this class is linked to a C-terminal region without the 5-phosphatase domain and the proline-rich region.

In this issue of Cell Research, Jing and his colleagues identified a novel Sac domain-containing protein, named as rat Sac3 (rSac3), when they searched for learning and memory-related functional genes [6]. This novel rSac3 gene showed a high degree of sequence similarity to human KIAA0274 (hSac3). The highly conserved catalytic sequence is RTNCVDCLDRTN in the sixth motif of the Sac domain of rSac3. Because its C-terminal does not contain the 5-phosphatase domain and the proline-rich region, rSac3 belongs to the second class of Sac domaincontaining proteins. This is consistent with the results that $\mathrm{rSac} 3$ has the ability of hydrolyzing phosphate of PtdIns(3)P, PtdIns(4)P, PtdIns(3,5)P2, but not PtdIns(5)P, PtdIns(4,5)P2, PtdIns(3,4,5)P3 [6].

The major cellular function of Sac domain-containing proteins is to modulate intracellular membrane trafficking, including exocytosis, endocytosis and membrane transportation between various intracellular compartments, via hydrolyzing their corresponding phosphoinositide substrates $[1,2,7,8]$. Synaptojanin 1 and 2 are involved in regulating endocytic vesicle fission and phosphoinositide dephosphorylation [1]. The rapid degradation of $\operatorname{PtdIns}(4,5) \mathrm{P} 2$ by synaptojanin 1 is of critical importance in the efficient synaptic vesicle regeneration and the recovery of normal presynaptic function after an exocytic burst [7]. Neurons of synaptojanin 1-deficient mice display an accumulation of clathrin-coated vesicles, indicating a defect in the late stage of uncoating process [7]. Although sharing the same substrate PtdIns(4,5)P2 with synaptojanin 1, synaptojanin 2 has different function from that of synaptojanin 1 [7]. Synaptojanin 2 plays a role at an early stage of endocytic pit formation. These results underscore the importance of a tight regulation of PtdIns(4,5)P2 levels at the membrane.

Sac1, the second class of Sac domain-containing proteins, is an ER- and Golgi-complex-associated phosphoinositide phosphatase. Sac1 may dephosphorylate PtdIns(4)P that spatially restricts to Golgi complex when PtdIns(4)P returns from the Golgi complex to the ER [1]. Fig4, another member of second class of Sac domain-containing proteins, is localized to the vacuolar membrane and is required for both generation and turnover of PtdIns(3,5)P2. Fig4 exhibits lipid phosphatase activity towards the 5-phosphate residue of PtdIns(3,5)P2. The pale tremor mouse with Fig4 mutation exhibits neurodegeneration [9]. Enlarged cytoplasmic vacuoles are filled in the cultured fibroblasts of the pale tremor mice [9].

The spatial strictness of rSac3 to ER, Golgi complex and recycling endosomes shown by Jing and colleagues suggests that this protein transports from the ER and Golgi complex, through the trans-Golgi network, to the intracellular vesicular trafficking compartments, and may play crucial roles in vesicular trafficking from the ER and Golgi complex to the plasma membrane [6]. This possibility led Jing and his colleagues to reveal the effects of rSac 3 on neurite growth. The nerve growth factor (NGF) stimulated differentiation of rat pheochromocytoma cell line PC12 cells into neuron-like cells with processes that extend to form long neurites [6]. Overexpression of rSac3 in PC12 cells promoted neurite outgrowth during NGF-stimulated neuronal differentiation [6]. However, inhibition of rSac3 expression results in reduced neurite outgrowth of NGFstimulated PC12 cells [6]. They further demonstrated that the role of $\mathrm{rSac} 3$ in promoting neurite outgrowth was attributed to the phosphoinositide phosphatase activity of rSac3.

Growing evidence suggests that neurite outgrowth and differentiation is mediated at least in part by PI3K signaling that facilitates both turning and branching at the growth cone or axon shaft in response to neurotrophin stimuli. In differentiating neurons activation of PI3K may occur at the growth cone. NGF stimulates the activation of PI3K which increases the levels of PtdIns $(3,4,5) \mathrm{P} 3$. The proline-rich inositol polyphosphate 5-phosphatase (PIPP) could hydrolyze PtdIns $(3,4,5) \mathrm{P} 3$ and regulate the activity of PI3K-dependent signaling [10]. Overexpression of PIPP significantly reduced the neurite extension in PC12 cells, and decreased PIPP protein expression was associated with significant hyperelongation of NGF differentiated neurites. These results suggest that PIPP functions as a negative regulator of PI3K signaling that promotes neurite elongation [10]. Interestingly, the phosphoinositide phosphatase rSac3 has completely opposite effects on neurite outgrowth in PC12 cells [6]. The difference is that $\mathrm{rSac} 3$ contains a Sac domain, whereas PIPP contains the 5-phosphatase domain and proline-rich region. PIPP plays roles in modulating neurite growth via hydrolyzing PtdIns $(3,4,5) \mathrm{P} 3$. However, the substrates of rSac3 does not include PtdIns $(3,4,5) \mathrm{P} 3$ [6]. These differences may explain why rSac3 and PIPP have the opposite effects on neurite outgrowth. Jing and his colleague found that rSac3 is localized in ER, Golgi complex and recycling endosomes. Because PtdIns(4)P is predominantly enriched at the Golgi complex [1], it is possible that the promoting effects of $\mathrm{rSac} 3$ on PC12 cell neurite extension may be mediated through hydrolyzation of PtdIns(4)P. Although the detail cellular and molecular mechanisms mediating the role of $\mathrm{rSac} 3$ in promoting neurite extension require further investigation, the identifi- 
cation of rSac3 would make significant contribution to our complete understanding of Sac domain-containing proteins and their cellular functions.

\section{References}

1. Di Paolo G, De Camilli P. Phosphoinositides in cell regulation and membrane dynamics. Nature 2006; 443:651-657.

2. Michell RH, Health VL, Lemmon MA, Dove SK. Phosphatidylinositol 3,5-bisphosphate: metabolism and cellular functions. Trends Biochem Sci 2006; 31:52-63.

3. Takenawa T, Itoh T. Phosphoinositides, key molecules for regulation of actin cytoskeletal organization and membrane traffic from the plasma membrane. Biochimica et Biophysica Acta 2001; 1533:190-206.

4. Hughes WE, Cooke FT, Parker PJ. Sac phosphatase domain proteins. Biochem J 2000; 350:337-352.

5. Ning K, Pei L, Liao M, et al. Dual Neuroprotective signaling mediated by downregulating two distinct phosphatase acitivities of PTEN. J Neurosci 2004; 24:4052-4060.

6. Yuan Y, Gao X, Guo N, et al. rSac, a novel Sac domain phosphoinositide phosphatase, promotes neurite outgrowth in PC12 cells. Cell Res 2007; 17:919-932.

7. Rusk N, Le PU, Mariggio S, et al. Syanptojanin 2 functions at an early step of clathrin-mediated endocytosis. Curr Biol 2003; 13:659-663.

8. Duex JE, Nau JJ, Kauffman EJ, Weisman LS. Phosphoinositide 5-phosphatase Fig4p is required for both acute rise and subsequent fall in stress-induced phosphatidylinositol 3,5-bisphosphate levels. Eukaryot Cell 2006; 5:723-731.

9. Chow CY, Zhang T, Dowling JJ, et al. Mutation of Fig4 causes neurodegeneration in the pale tremor mouse and patients with CMT4J. Nature 2007; 448:68-73.

10. Ooms LM, Fedele CG, Astle MV, et al. The inositol polyphosphate 5-phosphatase, PIPP, Is a novel regulator of phosphoinositide 3-kinase-dependent neurite elongation. Mol Biol Cell 2006; 17:607-622. 